EPJ Web of Conferences 59, 06002 (2013)

DOI: $10.1051 /$ epjconf/20135906002

(C) Owned by the authors, published by EDP Sciences, 2013

\title{
Development of our laser fusion integration simulation
}

\author{
Jinghong Lia ${ }^{a}$, Chuanlei Zhai, Shuanggui Li, Xin Li, Wudi Zheng, Heng Yong, \\ Qinghong Zeng, Xudeng Hang, Jin Qi, Rong Yang, Juan Cheng, Peng Song, \\ Peijun Gu, Aiqing Zhang, Hengbin An, Xiaowen Xu, Hong Guo, Xiaolin Cao, \\ Zeyao Mo, Wenbing Pei, Song Jiang and Shao-ping Zhu
}

Institute of Applied Physics and Computational Mathematics, PO Box 8009, Beijing 100094, PR China

\begin{abstract}
In the target design of the Inertial Confinement Fusion (ICF) program, it is common practice to apply radiation hydrodynamics code to study the key physical processes happening in ICF process, such as hohlraum physics, radiation drive symmetry, capsule implosion physics in the radiation-drive approach of ICF. Recently, many efforts have been done to develop our 2D integrated simulation capability of laser fusion with a variety of optional physical models and numerical methods. In order to effectively integrate the existing codes and to facilitate the development of new codes, we are developing an object-oriented structured-mesh parallel code-supporting infrastructure, called JASMIN. Based on two-dimensional three-temperature hohlraum physics code LARED-H and two-dimensional multi-group radiative transfer code LARED-R, we develop a new generation two-dimensional laser fusion code under the JASMIN infrastructure, which enable us to simulate the whole process of laser fusion from the laser beams' entrance into the hohlraum to the end of implosion. In this paper, we will give a brief description of our new-generation two-dimensional laser fusion code, named LARED-Integration, especially in its physical models, and present some simulation results of holhraum.
\end{abstract}

\section{INTRODUCTION}

Inertial confinement fusion (ICF) is an approach to fusion that relies on the inertia of the fuel mass to provide confinement [1]. Laser ICF is one way to fusion energy on the Earth, which uses a high power laser as a driver to implode capsules filled with fusion fuel and to produce thermonuclear energy. The capsule implosion is driven by either direct laser ablation or radiation ablation. In direct drive ICF, the laser beams are aimed directly at the capsule. In indirect drive ICF, laser energy is first converted into $\mathrm{x}$-ray radiation energy inside a high- $\mathrm{Z}$ enclosure, called hohlraum. The resulting $\mathrm{x}$-ray radiation implodes the spherical capsule fuel assembly mounted in the center of the hohlraum, bringing the central fuel to fusion conditions.

The largest laser facility in the world, the National Ignition Facility (NIF) housed at the Department of Energy's Lawrence Livermore National Laboratory, was formally completed in 2009 [2]. NIF is expected to allow scientists to achieve fusion ignition in the laboratory, obtaining more energy from the target than is provided by the laser. The completion of NIF opens the door to scientific advancement and discovery, and will lead to new breakthroughs in the worlds of astrophysics, materials science and many other scientific disciplines. Recently, the first integrated experiment at NIF was successful. With the completion of this test, NIF is beginning its next phase of the campaign to culminate in fusion ignition tests [3].

\footnotetext{
ae-mail: li_jinghong@iapcm.ac.cnr
}

This is an Open Access article distributed under the terms of the Creative Commons Attribution License 2.0, which permits unrestricted use, distribution, and reproduction in any medium, provided the original work is properly cited. 
ICF is complex, and numerical simulations can help us understand what happens in a small hohlraum [4]. Laser ICF research in China, started late in 1970s, is now organized by the National High-Tech Inertial Confinement Fusion Committee. Theory and simulation research on laser fusion is one important parts, and is mainly carried out at the Institute of Applied Physics and Computational Mathematics (IAPCM). We have been developing two-dimensional (2D) and three-dimensional (3D) simulation codes for laser fusion since the middle of 1990s. A system of 2D simulation based on LARED family of codes for the key physics issues of laser fusion was preliminarily built about ten years later [5].

\section{OUR LARED-INTEGRATION CODE}

With the rapid development of high performance computers, we need to develop ICF simulation codes to meet the requirement for large-scale simulation of laser fusion research.

The difficulty to understand laser ICF physics comes from the fact that many physical processes, such as hydrodynamics, radiative transfer, and Non-LTE atomic physics, occur simultaneously. The great challenges for laser ICF simulation come from: multi-level parallel programming, load imbalance, fast algorithms implementation, code complexity, and data visualization. An easy way to use supercomputer efficiently for large-scale high performance simulation is based on an objectoriented parallel code-supporting infrastructure. JASMIN (J Adaptive Structured Mesh applications Infrastructure) has been developed for high performance computation of multi-physics at IAPCM since 2004 [6]. The infrastructure includes common components independent of the problem, such as data management and communication, parallel, adaptive and visualization techniques, etc., and mature and standard algorithm. It supports large scale parallel simulations on adaptive structured mesh using massively parallel processing machines. Now, JASMIN has been developed to version 2.0, and finds many applications. It essentially meets the requirement for laser ICF codes.

In order to make use of the existing codes, we need to assemble LARED-H, LARED-R and LARED-I codes [5] under JASMIN into a new code, named LARED-integration. It has a variety of optional physical models and numerical methods. LARED-H is a two-dimensional non-LTE radiation hydrodynamic code for laser-target coupling and hohlraum physics modeling. Using the hohlraum radiation drive provided by the LARED-H calculation, we can perform simulations on capsule implosion and other application problems, such as hydrodynamic instability, radiation flux, opacity, and equation of state (EOS). RDMG is a 1D radiation hydrodynamic code with the radiation treated by models of transport, diffusion, and conduction. This code is used for research of problems such as lasertarget coupling, capsule implosion, radiation transport, radiation ablation, and radiation-driven shock. LARED-R is a 2D multi-group radiation diffusion-transport hydrodynamic code.

Benefiting from JASMIN's strong support of non-structured multi-block mesh for complicated geometry, we extend LARED-H code's ability to deal with large deformation. Now, LARED-H code achieved a whole simulation of NIF ignition-like target by allowing mesh across material interface.

LARED-Integration code will become our key 2-D physics code for studying laser indirectdrive ICF. Now, we give a brief description of its seven main modules shown in Fig 1: laser light, hydrodnamics, electron, ion, radiation, atomic physics, and burn products. 3-D ray-tracing technique is used for laser light propagation and absorption. Hydrodynamics (2-D) calculation relies on Lagrangian method on quadrilateral grid and Arbitrary Lagrange Eulerian (ALE) method. We use flux-limited thermal conduction for electrons and ions, and use several methods for radiation modeling: thermal conduction, multi-group flux-limited diffusion [7], multi-group transfer (discrete ordinate $\mathrm{S}_{N}$ ) [8]. Atomic physics includes average atom modeling, tabulated opacity (LTE), and tabulated EOS (LTE). Burn product uses local deposition. In the future, we plan to add Monte Carlo method for radiation modeling and charged particle transfer for burn product modeling. Compared with LARED-H code, LARED-Integration code enhances physical modeling related to radiation. 


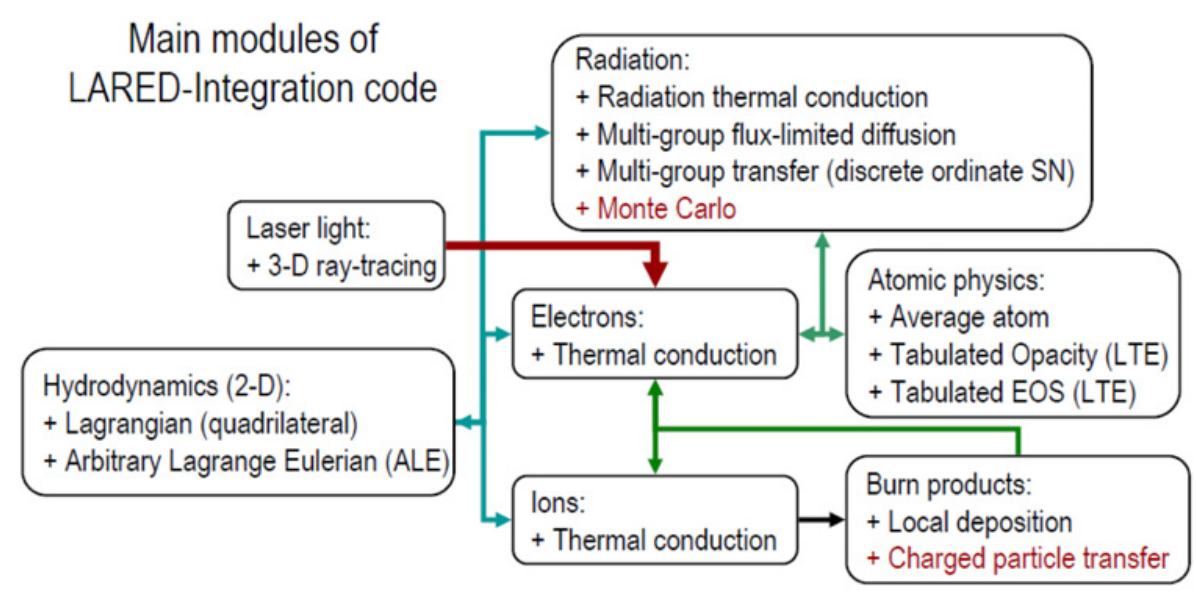

Figure 1. Main modules of LARED-Integration code. The two parts to be installed in the future are highlighted in red.

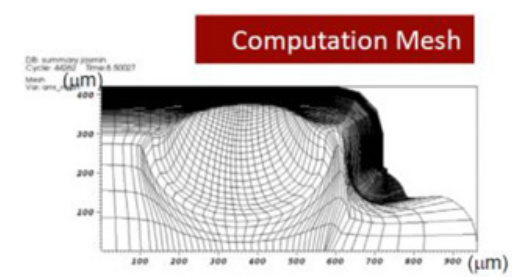

Electron temperature

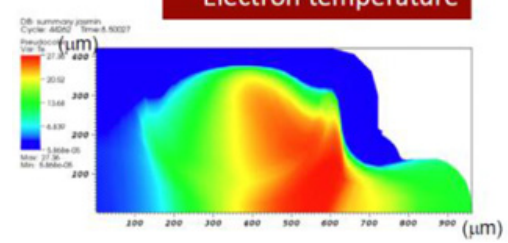

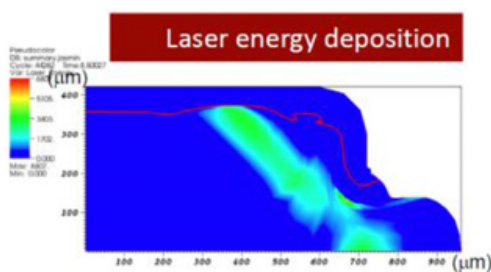

Radiation temperature

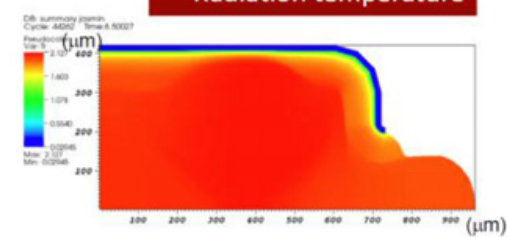

Figure 2. 64-group diffusion results of ICF hohlraum with LARED-Integration code.

\section{SOME SIMULATION RESULTS}

LARED-Integration project started in September 2009. Preliminary 3-temperature results of LLNL NIF ignition target with LARED-Integration increase our confidence.

After successfully passing a benchmark test for 1D laser gold planar target with simulation results of 1D RDMG code, LARED-Integration began multi-group radiation diffusion simulation. 64-group diffusion results of hohlraum are shown in Fig 2. By comparison, we can find that there are some small differences on the computation mesh movement, the distributions of electron and ion temperatures, laser deposition energy, and effective radiation temperature in one-group and 64-group radiation diffusion simulations.

Physical quantities for detailed description of hohlraum radiation environment are: radiation temperature, radiation flux, radiation spectrum, and radiation directionality. Multi-group radiative transfer calculation can provide all four physical quantities, while multi-group radiation diffusion calculation can provide the first three ones. However, with the help of post-processing package, we can obtain the information on radiation directionality by using multi-group diffusion simulation results. 

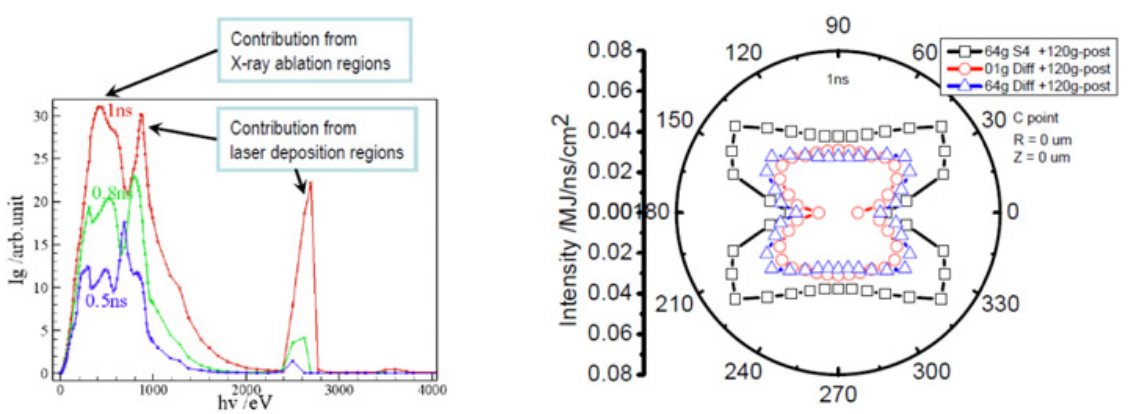

Figure 3. Some simulation results. Left: 64-group $S_{4}$ result of hohlraum radiation spectrum at different times. Right: Radiation angular distribution comparison for the cases of 64-group $\mathrm{S}_{4}$, 64-group diffusion and one-group diffusion.

Multi-group radiation transport result of radiation spectrum distribution is shown in Fig. 3 (left), and angular distribution of radiation intensity at hohlraum center is shown in Fig. 3 (right). The radiation intensity coming from laser deposition region, laser entrance hole, and other region are quite different. Multi-group radiation diffusion calculation and three-temperature model do not show strong radiation anisotropy as multi-group radiative tranfer calculation. Multi-group radiative tranfer calculation should be used for radiation directionality study.

\section{SUMMARY}

Based on LARED-H and LARED-R codes, we develop a new generation two-dimensional laser fusion code under the JASMIN infrastructure. Now, we have completed the multi-group version of LAREDIntegration code for studying indirect-drive ICF. Some simulation results on multi-group radiation transfer and diffusion of ICF hohlraum with LARED-Integration code are presented.

LARED-Integration is a culmination of more than two decades of ICF code development at IAPCM. We have developed LARED-Integration code for ICF 2D hohlraum design. Multi-group radiative transfer enhances our confidence in hohlaum radiation environment simulation. Multi-group radiation diffusion calculation also can provide hohlraum radiation with a higher precision than three-temperature model.

This work was supported by the National Hi-Tech Inertial Confinement Fusion Committee of China and National Natural Science Foundation of China (Project No. 91130002).

\section{References}

[1] J. D. Lindl, Phys. Plasmas 2, 3933 (1995); J. D. Lindl, et al., Phys. Plasmas 11, 339 (2004)

[2] http://www.energy.gov/news2009/

[3] https://lasers.llnl.gov/newsroom/project_status/index.php

[4] D. S. Clark, et al., Phys. Plasmas 15, 056305 (2008)

[5] W. B. Pei, Commun. Comput. Phys. 2, 255 (2007) ; S. Fu, et al., Chinese J. Numer. Math. Appl. 25, 275 (2004) ; Q. Zeng, et al., Competence in High Performance Computing 2010, 227 (2011)

[6] http://www.iapcm.ac.cn/jasmin; Z. Y. Mo, et al., Front. Comput. Sci. China. 4, 480 (2010)

[7] D. S. Kershaw, J. Comp. Phys. 39, 375 (1981)

[8] M. L. Adams and E. W. Larsen, Prog. Nucl. Energy 40, 3 (2002) 